\title{
A Computationally Efficient and Robust Implementation of the Continuous-Discrete Extended Kalman Filter
}

\author{
Jørgensen, John Bagterp; Thomsen, Per Grove; Madsen, Henrik; Kristensen, Morten Rode
}

Published in:

American Control Conference, 2007. ACC '07

Link to article, DOI:

10.1109/ACC.2007.4282549

Publication date:

2007

Document Version

Publisher's PDF, also known as Version of record

Link back to DTU Orbit

Citation (APA):

Jørgensen, J. B., Thomsen, P. G., Madsen, H., \& Kristensen, M. R. (2007). A Computationally Efficient and Robust Implementation of the Continuous-Discrete Extended Kalman Filter. In American Control Conference, 2007. ACC '07 IEEE. https://doi.org/10.1109/ACC.2007.4282549

\section{General rights}

Copyright and moral rights for the publications made accessible in the public portal are retained by the authors and/or other copyright owners and it is a condition of accessing publications that users recognise and abide by the legal requirements associated with these rights.

- Users may download and print one copy of any publication from the public portal for the purpose of private study or research.

- You may not further distribute the material or use it for any profit-making activity or commercial gain

- You may freely distribute the URL identifying the publication in the public portal 


\section{A Computationally Efficient and Robust Implementation of the Continuous-Discrete Extended Kalman Filter}

\author{
John Bagterp Jørgensen*, Per Grove Thomsen, Henrik Madsen \\ Informatics and Mathematical Modelling \\ Technical University of Denmark \\ DK-2800 Kgs. Lyngby, Denmark \\ $\{j b j$, pgt, hm\}@imm.dtu.dk
}

\author{
Morten Rode Kristensen \\ Department of Chemical Engineering \\ Technical University of Denmark \\ DK-2800 Kgs. Lyngby, Denmark \\ mrkekt.dtu.dk
}

\begin{abstract}
We present a novel numerically robust and computationally efficient extended Kalman filter for state estimation in nonlinear continuous-discrete stochastic systems. The resulting differential equations for the mean-covariance evolution of the nonlinear stochastic continuous-discrete time systems are solved efficiently using an ESDIRK integrator with sensitivity analysis capabilities. This ESDIRK integrator for the meancovariance evolution is implemented as part of an extended Kalman filter and tested on a PDE system. For moderate to large sized systems, the ESDIRK based extended Kalman filter for nonlinear stochastic continuous-discrete time systems is more than two orders of magnitude faster than a conventional implementation. This is of significance in nonlinear model predictive control applications, statistical process monitoring as well as grey-box modelling of systems described by stochastic differential equations.
\end{abstract}

\section{INTRODUCTION}

The objective of state estimation is to reconstruct the state of a system from process measurements given a model. State estimation has important applications in nonlinear model predictive control as well as in monitoring, prediction and fault detection. Several approaches to state estimation in systems modelled by ordinary differential equations exist. They include a rigorous probabilistic method solving Kolmogorov's (Fokker-Planck's) forward equation [1], [2] as well as approximative methods such as extended Kalman filtering (EKF) [3], [4] and optimization based approaches usually referred to as moving horizon estimation (MHE) [5]-[7]. The probabilistic approach based on solution of Kolmogorov's forward equation is applicable only to the simplest systems due to its requirement for solution of partial differential equations with the number of independent variables equal to the number of stochastic states. Moving horizon estimation has gained some popularity recently due to its similarity to model predictive control and its ability to handle constraints on the states and the stochastic process disturbances. While moving horizon estimation has a number of desirable properties compared to for instance extended Kalman filtering [8], its application in relation to model identification and in particular systematic rigorous grey-box model identification [9]-[11] seems problematic due to the computational demanding optimization needed for each state estimate. Grey-box identification for moving horizon estimation would give rise to several nested layers of optimization yielding a computational infeasible procedure. Undoubtedly, the extended Kalman filter is the most widely adopted state estimation technology for nonlinear systems and remains the standard technology for state estimation in nonlinear model predictive control applications despite recent popularity of moving horizon estimation [4], [12]-[18]. Furthermore, systematic methods for grey-box identification of nonlinear models used in continuous-discrete time extended Kalman filters exist [9], [10], [19], [20].

This paper is organized as follows. Section II introduces the extended Kalman filter for continuous-discrete stochastic systems and presents an efficient numerical algorithm. Section III demonstrates the performance of the new EKF algorithm based on scalable PDE system, while Section IV provides the conclusions.

\section{NUMERICAL IMPLEMENTATION}

In this section, we develop a robust yet efficient numerical procedure for filtering and one-step ahead prediction by the extended Kalman filter for the continuous-discrete stochastic system [1], [21], [22]

$$
\begin{aligned}
& d \boldsymbol{x}(t)=f(t, \boldsymbol{x}(t)) d t+\sigma d \boldsymbol{\omega}(t) \\
& \boldsymbol{y}\left(t_{k}\right)=h\left(t_{k}, \boldsymbol{x}\left(t_{k}\right)\right)+\boldsymbol{v}_{k}
\end{aligned}
$$

in which $\boldsymbol{\omega}(t): t \geq 0$ is a standard Wiener process and $\sigma$ is a time invariant matrix. $\sigma$ is time-invariant in many practical problems [9], [23]. For this reason and due to numerical efficiency considerations, we specialize to the case with $\sigma(t)=\sigma$ being time-invariant. The measurement noise is normally distributed, $\boldsymbol{v}_{k} \sim N\left(0, R_{k}\right)$, and the initial state is a realization of a random variable with finite first and second moment, i.e. $\boldsymbol{x}_{0} \sim \mathbb{F}\left(\hat{x}_{0 \mid-1}, P_{0 \mid-1}\right)$.

The major operation in the continuous-discrete extended Kalman filter concerns integration of the mean-covariance pair

$$
\begin{aligned}
& \frac{d \hat{x}_{k}(t)}{d t}=f\left(t, \hat{x}_{k}(t)\right) \\
& \frac{d P_{k}(t)}{d t}=A(t) P_{k}(t)+P_{k}(t) A(t)^{\prime}+\sigma \sigma^{\prime}
\end{aligned}
$$

with

$$
A(t)=\frac{\partial f}{\partial x}\left(t, \hat{x}_{k}(t)\right)
$$


and initial conditions

$$
\begin{aligned}
\hat{x}_{k}\left(t_{k}\right) & =\hat{x}_{k \mid k} \\
P_{k}\left(t_{k}\right) & =P_{k \mid k}
\end{aligned}
$$

The mean-covariance pair defined by (2) is solved numerically by solution of

$$
\begin{aligned}
\frac{d \hat{x}_{k}(t)}{d t}=f\left(t, \hat{x}_{k}(t)\right) & \hat{x}_{k}\left(t_{k}\right)=\hat{x}_{k \mid k} \\
\frac{d \Phi(t, s)}{d t}=A(t) \Phi(t, s) & \Phi(s, s)=I
\end{aligned}
$$

in which

$$
A(t)=\frac{\partial f}{\partial x}\left(t, \hat{x}_{k}(t)\right)
$$

and

$$
\begin{aligned}
P_{k}(t)=\Phi & \left(t, t_{k}\right) P_{k \mid k} \Phi\left(t, t_{k}\right)^{\prime} \\
& +\int_{t_{k}}^{t} \Phi(t, s) \sigma \sigma^{\prime} \Phi(t, s)^{\prime} d s
\end{aligned}
$$

In the implemented algorithm the matrix square root, $P_{k}(t)^{1 / 2}$, rather than $P_{k}(t)$ itself is computed.

A numerical procedure for solution of (3) based on the ESDIRK algorithm is presented [24], [25]. The ESDIRK algorithm is an explicit singly diagonal implicit Runge-Kutta method. The implementation is equipped with an adaptive step size controller such that the computed solution satisfies certain accuracy specifications. The method is constructed to be able to handle stiff systems and it is a single-step method. In contrast to multi-step methods e.g. BDF methods, singlestep mehtods are ideally suited for systems with frequent discontinuities [25], [26]. For computer controlled systems, discontinuities typically arise at each sample time because the manipulated process inputs are adjusted. In addition, the ESDIRK algorithm is simple to implement and easy to modify. This implies that the ESDIRK algorithm may be used to solve (3) for $\hat{x}_{k}\left(t_{k+1}\right)$ and $P_{k}\left(t_{k+1}\right)^{1 / 2}$ in a way that is not much more computational expensive than solving (3a) for $\hat{x}_{k}\left(t_{k+1}\right)$.

\section{A. The ESDIRK Method}

The ESDIRK method is described in [25]. Here, an outline of the algorithm for solution of (3a) is provided. At each internal integration step chosen by the step-size controller, the following Runge-Kutta equations are solved

$$
\begin{array}{rlrl}
T_{i} & =t_{n}+c_{i} h & i=1,2,3,4 \\
X_{i} & =x_{n}+h \sum_{j=1}^{i} a_{i j} f\left(T_{i}, X_{i}\right) & i=1,2,3,4 \\
x_{n+1} & =x_{n}+h \sum_{i=1}^{4} b_{i} f\left(T_{i}, X_{i}\right) & \\
e_{n+1} & =h \sum_{i=1}^{4} d_{i} f\left(T_{i}, X_{i}\right)
\end{array}
$$

in which the coefficients of ESDIRK methods are described by the Butcher tableau

$$
\begin{array}{c|cccc}
0 & 0 & & & \\
c_{2} & a_{21} & \gamma & & \\
c_{3} & a_{31} & a_{32} & \gamma & \\
1 & b_{1} & b_{2} & b_{3} & \gamma \\
\hline x_{n+1} & b_{1} & b_{2} & b_{3} & \gamma \\
\hline e_{n+1} & d_{1} & d_{2} & d_{3} & d_{4}
\end{array}=\begin{array}{c|c}
\mathbf{c} & \mathcal{A} \\
\hline & \mathbf{b}^{T} \\
\hline & \mathbf{d}^{T}
\end{array}
$$

$e_{n+1}$ is an estimated error of the solution. This estimated error is used by the step-length controller to adjust the step size, $h$. The main numerical effort for each integration step concerns solution of (4b). (4b) may be stated as the nonlinear system of equations

$$
R_{i}\left(T_{i}, X_{i}\right)=0 \quad i=1,2,3,4
$$

with the residual, $R_{i}\left(T_{i}, X_{i}\right)$, defined as

$$
\begin{aligned}
R_{i}\left(T_{i}, X_{i}\right) & =X_{i}-x_{n}-h \sum_{j=1}^{i} a_{i j} f\left(T_{j}, X_{j}\right) \\
& =X_{i}-h \gamma f\left(T_{i}, X_{i}\right)-x_{n}-\sum_{j=1}^{i-1} h a_{i j} f\left(T_{j}, X_{j}\right)
\end{aligned}
$$

It should be noted, that (6) needs to be solved for $i=2,3,4$ only, as $X_{1}=x_{n}$. In addition, as a consequence of the diagonal structure of the A-matrix in the Butcher tableau for ESDIRK methods, $X_{i}$ with $i=2,3,4$ can be computed successively rather than simultaneously. (6) is solved by Newton's method, i.e.

$$
\begin{aligned}
& M \Delta X_{i}=-R_{i}\left(T_{i}, X_{i}^{(l)}\right) \\
& X_{i}^{(l+1)}=X_{i}^{(l)}+\Delta X_{i}
\end{aligned}
$$

in which the iteration matrix, $M$, is defined by

$$
M=I-h \gamma A \quad A=\frac{\partial f}{\partial x}\left(t_{n}, x_{n}\right)
$$

In computational efficient ESDIRK algorithms for integration of (3a) only, the iteration matrix, $M$, is evaluated and factorized adaptively and not at every integration interval. For the joint computation of the mean and state sensitivities, (3a)-(3b), by the staggered direct method [27], the iteration matrix, $M$, and the Jacobian, $A=\frac{\partial f}{\partial x}\left(t_{n}, x_{n}\right)$, are needed at every integration interval. Consequently, in the implementation considered in this paper, the iteration matrix is evaluated and factorized for each integration step, i.e. at $\left\{t_{n}, x\left(t_{n}\right)\right\}$.

\section{B. State Sensitivities and Covariance}

In the solution of (3b), we invoke the following assumption Assumption 1 (Constant Jacobian Matrix): The Jacobian matrix

$$
A(t)=A=\frac{\partial f}{\partial x}\left(t_{n}, x\left(t_{n}\right)\right) \quad t \in\left[t_{n}, t_{n+1}=t_{n}+h\right]
$$

is constant in each integration interval, $\left[t_{n}, t_{n+1}=t_{n}+h\right]$, accepted by the step-length controller. 
Numerical experience has demonstrated that this is an excellent assumption, because the step-length controller reduces the step length in regions where the solution changes unpredictably, i.e. in regions in which $A(t)$ is not constant. Hence, due to the adjusted step-length, $h$, the Jacobian, $A(t)=A$, as well as the iteration matrix, $M=I-h \gamma A$, may be assumed to be constant in all integration intervals accepted by the step-length control algorithm.

By Assumption 1, (3b) may be written as

$$
\frac{d \Phi(t, s)}{d t}=A \Phi(t, s) \quad \Phi(s, s)=I
$$

which for time invariant matrices, $A$, has the solution [28]

$$
\Phi(t, s)=\exp [A(t-s)]
$$

In addition, due to the time invariance in the interval $\left[t_{n}, t_{n+1}\right]$, the state transition matrix, $\Phi(t, s)$, may be expressed as

$$
\begin{aligned}
\Phi(t, s) & =\Phi(t-s, 0) \\
& =\exp [A(t-s)]=\exp (A \tau)=\Phi(\tau)
\end{aligned}
$$

in which $\tau=t-s$. This implies

$$
\begin{aligned}
& \int_{t_{n}}^{t_{n+1}} \Phi\left(t_{n+1}, s\right) \sigma \sigma^{\prime} \Phi\left(t_{n+1}, s\right)^{\prime} d s= \\
& \int_{t_{n}}^{t_{n+1}} \Phi\left(t_{n+1}-s\right) \sigma \sigma^{\prime} \Phi\left(t_{n+1}-s\right)^{\prime} d s= \\
& -\int_{\tau=t_{n+1}-t_{n}}^{\tau=0} \Phi(\tau) \sigma \sigma^{\prime} \Phi(\tau)^{\prime} d \tau=\int_{0}^{h} \Phi(\tau) \sigma \sigma^{\prime} \Phi(\tau)^{\prime} d \tau
\end{aligned}
$$

in which $\tau=t_{n+1}-s$. For illustration, assume that $P_{n}=$ $P_{k \mid k}$ and $t_{k}=t_{n}$. Then, using $\Phi\left(t, t_{k}\right)=\Phi\left(t, t_{n}\right)=$ $\Phi\left(t, t_{n+1}\right) \Phi\left(t_{n+1}, t_{n}\right)$, we obtain

$$
\begin{aligned}
P_{k}(t)= & \Phi\left(t, t_{k}\right) P_{k \mid k} \Phi\left(t, t_{k}\right)^{\prime}+\int_{t_{k}}^{t} \Phi(t, s) \sigma \sigma^{\prime} \Phi(t, s)^{\prime} d t \\
= & \Phi\left(t, t_{n+1}\right) P_{n+1} \Phi\left(t, t_{n+1}\right)^{\prime} \\
& +\int_{t_{n+1}}^{t} \Phi(t, s) \sigma \sigma^{\prime} \Phi(t, s) d s
\end{aligned}
$$

in which

$$
\begin{aligned}
P_{n+1}= & \Phi\left(t_{n+1}, t_{n}\right) P_{n} \Phi\left(t_{n+1}, t_{n}\right)^{\prime} \\
& +\int_{t_{n}}^{t_{n+1}} \Phi\left(t_{n+1}, s\right) \sigma \sigma^{\prime} \Phi\left(t_{n+1}, s\right)^{\prime} d s \\
= & \Phi(h) P_{n} \Phi(h)^{\prime}+\int_{0}^{h} \Phi(\tau) \sigma \sigma^{\prime} \Phi(\tau)^{\prime} d \tau
\end{aligned}
$$

Consequently, for each integration interval, $\left[t_{n}, t_{n+1}=\right.$ $\left.t_{n}+h\right]$, accepted by the step-length control algorithm we may under Assumption 1 propagate the covariance matrix $P_{n}=P\left(t_{n}\right)=P_{k}\left(t_{n}\right)$ to the covariance matrix $P_{n+1}=$ $P\left(t_{n+1}\right)=P_{k}\left(t_{n+1}\right)$ by solution of

$$
\begin{aligned}
& \frac{d \Phi(\tau)}{d \tau}=A \Phi(\tau) \quad \Phi(0)=I \\
& P_{n+1}=\Phi(h) P_{n} \Phi(h)^{\prime}+\int_{0}^{h} \Phi(\tau) \sigma \sigma^{\prime} \Phi(\tau)^{\prime} d \tau
\end{aligned}
$$

in which $A=\frac{\partial f}{\partial x}\left(t_{n}, x\left(t_{n}\right)\right)$. (16a) is the sensitivity equation and may be solved in the quadrature points using the ESDIRK integration scheme, i.e.

$$
\begin{aligned}
& \Phi_{1}=I \\
& \Phi_{2}=M^{-1}\left(I+h a_{21} A\right) \\
& \Phi_{3}=M^{-1}\left(I+A\left(h a_{31} I+h a_{32} \Phi_{2}\right)\right) \\
& \Phi_{4}=M^{-1}\left(I+A\left(h a_{41} I+h a_{42} \Phi_{2}+h a_{43} \Phi_{3}\right)\right)
\end{aligned}
$$

in which $\Phi_{i}=\Phi\left(\tau_{i}\right)$ and $\tau_{i}=c_{i} h$. Note that the iteration matrix, $M=I-h \gamma A$, is already factorized and this factorization may be reused. Subsequently, (16b) is solved by the quadrature formula of ESDIRK, i.e.

$$
\begin{aligned}
P_{n+1} & =\Phi(h) P_{n} \Phi(h)^{\prime}+\int_{0}^{h} \Phi(\tau) \sigma \sigma^{\prime} \Phi(\tau)^{\prime} d \tau \\
& \approx \Phi_{4} P_{n} \Phi_{4}^{\prime}+\sum_{i=1}^{4} h b_{i} \Phi_{i} \sigma \sigma^{\prime} \Phi_{i}^{\prime} \\
& =\Phi_{4} P_{n} \Phi_{4}^{\prime}+h b_{1} \sigma \sigma^{\prime}+\sum_{i=2}^{4} h b_{i} \Phi_{i} \sigma \sigma^{\prime} \Phi_{i}^{\prime}
\end{aligned}
$$

Let $Q=\sigma \sigma^{\prime}=Q^{1 / 2} Q^{T / 2}$ in which $Q^{1 / 2}$ is the matrix square root of $Q$. The matrix square root is a lower triangular matrix. The square root, $P_{n}^{1 / 2}$, may be propagated into the square root, $P_{n+1}^{1 / 2}$, by the following sequence of orthogonal transformations [4]

$$
\begin{aligned}
& {\left[\begin{array}{ll}
\Phi_{4} P_{n}^{1 / 2} & \sqrt{h b_{1}} Q^{1 / 2}
\end{array}\right] \Theta_{1}=\left[\begin{array}{ll}
X^{1 / 2} & 0
\end{array}\right]} \\
& {\left[\begin{array}{ll}
X^{1 / 2} & \Phi_{2} \sqrt{h b_{2}} Q^{1 / 2}
\end{array}\right] \Theta_{2}=\left[\begin{array}{ll}
Y^{1 / 2} & 0
\end{array}\right]} \\
& {\left[\begin{array}{ll}
Y^{1 / 2} & \Phi_{3} \sqrt{h b_{3}} Q^{1 / 2}
\end{array}\right] \Theta_{3}=\left[\begin{array}{ll}
Z^{1 / 2} & 0
\end{array}\right]} \\
& {\left[\begin{array}{ll}
Z^{1 / 2} & \Phi_{4} \sqrt{h b_{4}} Q^{1 / 2}
\end{array}\right] \Theta_{4}=\left[\begin{array}{ll}
P_{n+1}^{1 / 2} & 0
\end{array}\right]}
\end{aligned}
$$

\section{Integration Algorithm}

The ESDIRK implementation for solution of (3) and thereby solution of (2) is stated in Algorithm 1. The algorithm solves (3) in the time interval $\left[t_{k}, t_{k+1}\right]$. For systems with constant sample time, $T_{s}$, the final time is $t_{k+1}=$ $t_{k}+T_{s}$. However, the method is not restricted to systems with constant sampling time and this is emphasized by the general notation.

Based on the accuracy specifications, Algorithm 1 applies a step-length controller [25] to subdivide the time interval, $\left[t_{k}, t_{k+1}\right]$, into smaller intervals. A solution to (3a) is accepted when it meets the accuracy specifications. At each solution acceptance, the square root of the covariance is propagated using Algorithm 2. Algorithm 2 is based on Assumption 1 in the time subinterval $[t, t+h]$.

The ESDIRK algorithm for solution of (2) as well as (3) may be stated compactly as

$$
\begin{aligned}
& {\left[\hat{x}_{k+1 \mid k}, P_{k+1 \mid k}^{1 / 2}\right]=} \\
& \quad \operatorname{ESDDIRK}\left(t_{k}, t_{k+1}, \hat{x}_{k \mid k}, P_{k \mid k}^{1 / 2}, Q^{1 / 2}\right)
\end{aligned}
$$

and consists of Algorithm 1 and 2. The inputs to the algorithm is the initial and final time, $t_{k}$ and $t_{k+1}$, the initial 
state (filtered state estimate), $\hat{x}_{k}\left(t_{k}\right)=\hat{x}_{k \mid k}$, the matrix square root, $P_{k}\left(t_{k}\right)^{1 / 2}=P_{k \mid k}^{1 / 2}$, of the initial state covariance (filtered state covariance), and $Q^{1 / 2}$ defined by the relation $\sigma \sigma^{\prime}=Q^{1 / 2} Q^{T / 2}$. The outputs from the algorithm are the predicted state, $\hat{x}_{k+1 \mid k}=\hat{x}_{k}\left(t_{k+1}\right)$, and the matrix square root of the associated state covariance, $P_{k+1 \mid k}^{1 / 2}=$ $\left[P_{k}\left(t_{k+1}\right)\right]^{1 / 2}$.

\section{EKF Algorithm}

A numerical procedure using ESDIRK for the extended Kalman filter of the continuous-discrete system (1) is stated in Algorithm 3. The initialization of Algorithm 3 is stated in Algorithm 4. For numerical robustness, the algorithm propagates the matrix square roots of the involved covariance matrices [4]. The arrangement of the operations in some implementations may vary from the arrangement stated in Algorithm 3. The order of the filter and prediction operations is mainly dependent on the intended applications of the extended Kalman filter.

The particular continuous-discrete time extended Kalman filter defined by Algorithm 3 and 4 is stated for a general state estimation application with non-uniform arrival of measurements in mind. Though for notational simplicity it is not stated in the algorithm, the extended Kalman filter is implemented with possible missing observations indicated by a status flag on the measurement vector. Rows corresponding to non-valid measurements are simply removed from $y_{k}$, $\hat{y}_{k \mid k-1}$ and $C_{k}$. Columns and rows corresponding to missing observations are removed from $R_{k}$, and a lower triangular square root matrix of the result is computed. By allowing missing observations, the algorithm can quite easily be embedded in a procedure that handles delayed measurements, i.e. laboratory measurements.

For control applications with fixed sampling time, $T_{s}$, and no missing observations the order of the one-step ahead predictor and filter of the extended Kalman filter may be rearranged. In the control case, it is important to compute the state estimate and subsequently the control as fast as possible in order to minimize the computational delay. Based on stored values of $\left\{\hat{y}_{k \mid k-1}, R_{k \mid k-1}^{1 / 2}, \bar{K}_{f x, k}\right\}$, the filtered estimate, $\hat{x}_{k \mid k}$, is computed by a few matrix-vector operations as a new measurement, $y_{k}$, becomes available. The filtered state, $\hat{x}_{k \mid k}$, may then be used as the initial state in a nonlinear predictive control computation and the computed initial control implemented before the extended Kalman filter is updated by the computations in the one-step ahead prediction block.

In all cases, the implementation of the extended Kalman filter for continuous-discrete systems is simplified considerably by use of the ESDIRK algorithm which handles the real computational complexity of the continuous-discrete extended Kalman filter.

\section{EXAMPle: Adiabatic FiXed-Bed ReACtoR}

To illustrate the efficiency of the proposed extended Kalman filter algorithm, we consider a model of an adiabatic

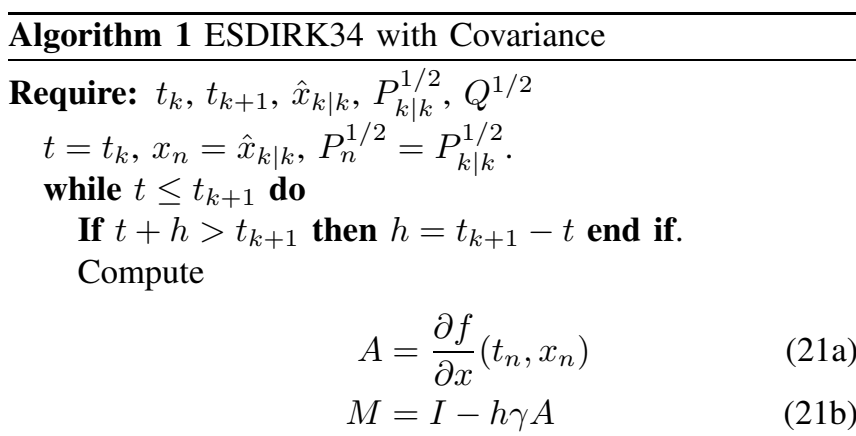

and $L U$-factorize $M$.

Compute the internal stages iteratively for $i=2,3,4$ using the $L U$-factorization of $M$ :

while $t o l \leq\|R\|$ do

Compute the residual vector $R$ and solve for $\Delta X_{i}$

$$
\begin{aligned}
R\left(X_{i}^{(l)}\right)=X_{i}^{(l)}-h \gamma f\left(T_{i}, X_{i}^{(l)}\right) & \\
& \quad-x_{n}-h \sum_{j=1}^{i-1} a_{i j} f_{j} \\
M \Delta X_{i}= & R\left(X_{i}^{(l)}\right) \\
X_{i}^{(l+1)}= & X_{i}^{(l)}-\Delta X_{i}
\end{aligned}
$$

end while

Compute the error estimate $e_{n+1}$ and tolerance monitor $r$ :

$$
\begin{aligned}
e_{n+1} & =\sum_{j=1}^{4} h d_{j} f_{j} \\
r & =\sqrt{\frac{1}{n_{s}} \sum_{i=1}^{n_{s}}\left(\frac{\left(e_{n+1}\right)_{i}}{\text { atol }_{i}+\left|\left(x_{n}\right)_{i}\right| \text { rtol }_{i}}\right)^{2}}
\end{aligned}
$$

if $r \leq 1$ then

Accept the step, update the time $t \leftarrow t+h$, and update the solution

$$
x_{n+1}=X_{4}
$$

Compute the covariance $P_{n+1}^{1 / 2}$ using Algorithm 2 and $P_{n}^{1 / 2}$ as initial condition.

Compute new step size $h$ using the error controller. else

Compute a new step size $h$ using the error controller. end if

end while

Return: $\hat{x}_{k+1 \mid k}=x_{n+1}, P_{k+1 \mid k}^{1 / 2}=P_{n+1}^{1 / 2}$ 


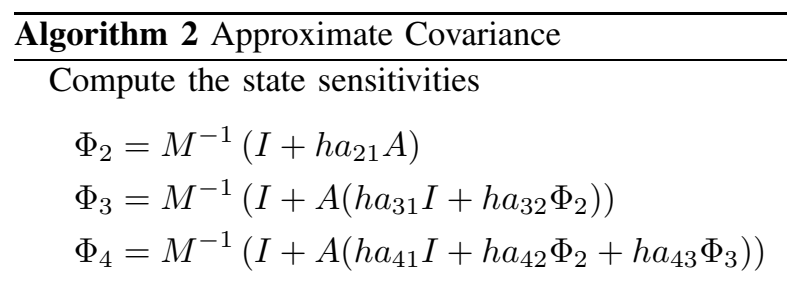

using the LU-factorization of $M$.

Compute square root matrices by orthogonal transformations (Householder operations)

$$
\begin{aligned}
& {\left[\begin{array}{ll}
X^{1 / 2} & 0
\end{array}\right] \leftarrow\left[\begin{array}{ll}
\Phi_{4} P_{n}^{1 / 2} & \sqrt{h b_{1}} Q^{1 / 2}
\end{array}\right] \Theta_{1}} \\
& {\left[\begin{array}{ll}
X^{1 / 2} & 0
\end{array}\right] \leftarrow\left[\begin{array}{ll}
X^{1 / 2} & \Phi_{2} \sqrt{h b_{2}} Q^{1 / 2}
\end{array}\right] \Theta_{2}} \\
& {\left[\begin{array}{ll}
X^{1 / 2} & 0
\end{array}\right] \leftarrow\left[\begin{array}{ll}
X^{1 / 2} & \Phi_{3} \sqrt{h b_{3}} Q^{1 / 2}
\end{array}\right] \Theta_{3}} \\
& {\left[\begin{array}{ll}
X^{1 / 2} & 0
\end{array}\right] \leftarrow\left[\begin{array}{ll}
X^{1 / 2} & \Phi_{4} \sqrt{h b_{4}} Q^{1 / 2}
\end{array}\right] \Theta_{4}}
\end{aligned}
$$

Set $P_{n+1}^{1 / 2}=X^{1 / 2}$. Return $P_{n+1}^{1 / 2}$.

fixed-bed reactor with a feed-effluent heat-exchanger. This model has been extensively used for bifurcation studies of distributed systems [29]. In this paper, the model is used to benchmark the EKF algorithm based on ESDIRK for different model orders resulting from the spatial discretization. Let $\alpha=\alpha(x, t)$ be the conversion of a reactant and $\theta=\theta(x, t)$ be the dimensionless temperature as function of position, $x$, in a fixed-bed reactor and time, $t$. Then the model of the fixed bed reactor consists of the system of partial differential equations

$$
\begin{aligned}
\varepsilon \frac{\partial \alpha}{\partial t} & =-\frac{\partial \alpha}{\partial x}+\frac{1}{P e_{m}} \frac{\partial^{2} \alpha}{\partial x^{2}}+\operatorname{DaR}(\alpha, \theta) \\
\frac{\partial \theta}{\partial t} & =-\frac{\partial \theta}{\partial x}+\frac{1}{P e_{h}} \frac{\partial^{2} \theta}{\partial x^{2}}+\operatorname{DaR}(\alpha, \theta)
\end{aligned}
$$

in which the reaction rate is given by

$$
R(\alpha, \theta)=(1-\alpha)^{r} \exp \left(\gamma \frac{\beta \theta}{1+\beta \theta}\right)
$$

The boundary conditions are

$$
\begin{aligned}
\alpha(0, t) & =\left.\frac{1}{P e_{m}} \frac{\partial \alpha}{\partial x}\right|_{x=0} \\
\theta(0, t) & =f \theta(1, t)+\left.\frac{1}{P e_{h}} \frac{\partial \theta}{\partial x}\right|_{x=0} \\
\left.\frac{\partial \alpha}{\partial x}\right|_{x=1} & =0 \\
\left.\frac{\partial \theta}{\partial x}\right|_{x=1} & =0
\end{aligned}
$$

and the initial conditions are some perturbation of the steadystate. The parameters are $\varepsilon=0.001, P e_{m}=P e_{h}=200$, $\gamma=15, r=2, \beta=0.4, f=0.3$, and $D a=0.1$.

The model (34) is reduced to a system of ordinary differential equations by employing an upwind discretization scheme for the convection terms, $\alpha_{x}$ and $\theta_{x}$, and a central difference discretization for the diffusion, $\alpha_{x x}$, and conduction, $\theta_{x x}$, terms. The boundary conditions at $x=0$ are discretized
Algorithm $3 \mathrm{EKF}$ for the continuous-discrete system (1)
Require: $t_{k-1}, t_{k}, \hat{x}_{k-1 \mid k-1}, P_{k-1 \mid k-1}^{1 / 2}, y_{k}, Q^{1 / 2}, R_{k}$
One-step ahead prediction:
Compute the one-step ahead predicted state, $\hat{x}_{k \mid k-1}$, and the square-root covariance, $P_{k \mid k-1}^{1 / 2}$, using Algorithm 1:

$$
\begin{aligned}
& {\left[\hat{x}_{k \mid k-1}, P_{k \mid k-1}^{1 / 2}\right]=} \\
& \quad \operatorname{ESD} \mathcal{I} \mathcal{R} \mathcal{K}\left(t_{k-1}, t_{k}, \hat{x}_{k-1 \mid k-1}, P_{k-1 \mid k-1}^{1 / 2}, Q^{1 / 2}\right)
\end{aligned}
$$

Compute the one-step ahead measurement prediction, $\hat{y}_{k \mid k-1}$, and the matrix $C_{k}$ :

$$
\begin{aligned}
\hat{y}_{k \mid k-1} & =h\left(t_{k}, \hat{x}_{k \mid k-1}\right) \\
C_{k} & =\frac{\partial h}{\partial x}\left(t_{k}, \hat{x}_{k \mid k-1}\right)
\end{aligned}
$$

Compute the measurement update matrices by an orthogonal transformation

$$
\left[\begin{array}{cc}
R_{k \mid k-1}^{1 / 2} & 0 \\
\bar{K}_{f x, k} & P_{k \mid k}^{1 / 2}
\end{array}\right] \leftarrow\left[\begin{array}{cc}
R_{k}^{1 / 2} & C_{k} P_{k \mid k-1}^{1 / 2} \\
0 & P_{k \mid k-1}^{1 / 2}
\end{array}\right] \Theta_{M}
$$

Filter:

Compute the filtered state

$$
\begin{aligned}
e_{k} & =y_{k}-\hat{y}_{k \mid k-1} \\
\bar{e}_{k} & =\left(R_{k \mid k-1}^{1 / 2}\right)^{-1} e_{k} \\
\hat{x}_{k \mid k} & =\hat{x}_{k \mid k-1}+\bar{K}_{f x, k} \bar{e}_{k}
\end{aligned}
$$

Return: $\hat{x}_{k \mid k}, P_{k \mid k}^{1 / 2}$

spatially using the Euler discretization. A fixed uniform mesh with grid size $\Delta x=1.0 /(N+1)$ is employed. The resulting number of ODEs describing the system is $2 N$. The steadystate is unstable and ends up in a limit cycle as shown in Figure 1 using $N=200$.

The only stochastic noise affecting the process is assumed to be variations in the inlet temperature, i.e. $\sigma=$ $e_{2}=\left[\begin{array}{lllll}0 & 1 & 0 & \ldots & 0\end{array}\right]^{\prime}$. The measurements used by the EKF are temperature measurements located at $x=$ $0.2,0.4,0.6,0.8$. All measurements have unit additive measurement noise, i.e. $R=I_{4,4}$. The mean of the initial distribution of the states is assumed to be equal to the actual states and the corresponding covariance matrix is a unit-matrix. The resulting system is a continuous-discrete stochastic system (1) with $2 N$ stochastic differential equations and 4 measurement equations. The dependence of the system size on $N$, allows comparative studies of various EKF implementations for medium- to large-scale systems. It should be noted that the model obtained is highly structured. This implies that for large $N$, it matters whether the integration is conducted using dense or sparse linear algebra. Therefore, we compare dense and sparse implementations for this example. Even though the Jacobian is sparse, the associated covariance matrix is not sparse. This implies that the covariance matrix computations in the ESDIRK based EKF are still dense. Consequently, for 


\section{Algorithm 4 EKF Initialization \\ Require: $\hat{x}_{0 \mid-1}, P_{0 \mid-1}^{1 / 2}, y_{0}, R_{0}$ \\ One-step ahead prediction:}

One-step ahead measurement prediction:

$$
\begin{aligned}
\hat{y}_{0 \mid-1} & =h\left(t_{0}, \hat{x}_{0 \mid-1}\right) \\
C_{0} & =\frac{\partial h}{\partial x}\left(t_{0}, \hat{x}_{0 \mid-1}\right)
\end{aligned}
$$

Compute the measurement update matrices by an orthogonal transformation

$$
\left[\begin{array}{cc}
R_{0 \mid-1}^{1 / 2} & 0 \\
\bar{K}_{f x, 0} & P_{0 \mid 0}^{1 / 2}
\end{array}\right] \leftarrow\left[\begin{array}{cc}
R_{0}^{1 / 2} & C_{0} P_{0 \mid-1}^{1 / 2} \\
0 & P_{0 \mid-1}^{1 / 2}
\end{array}\right] \Theta_{M}
$$

Filter:

Compute the filtered state

$$
\begin{aligned}
e_{0} & =y_{0}-\hat{y}_{0 \mid-1} \\
\bar{e}_{0} & =\left(R_{0 \mid-1}^{1 / 2}\right)^{-1} e_{0} \\
\hat{x}_{0 \mid 0} & =\hat{x}_{0 \mid-1}+\bar{K}_{f x, 0} \bar{e}_{0}
\end{aligned}
$$

Return: $\hat{x}_{0 \mid 0}, P_{0 \mid 0}^{1 / 2}$
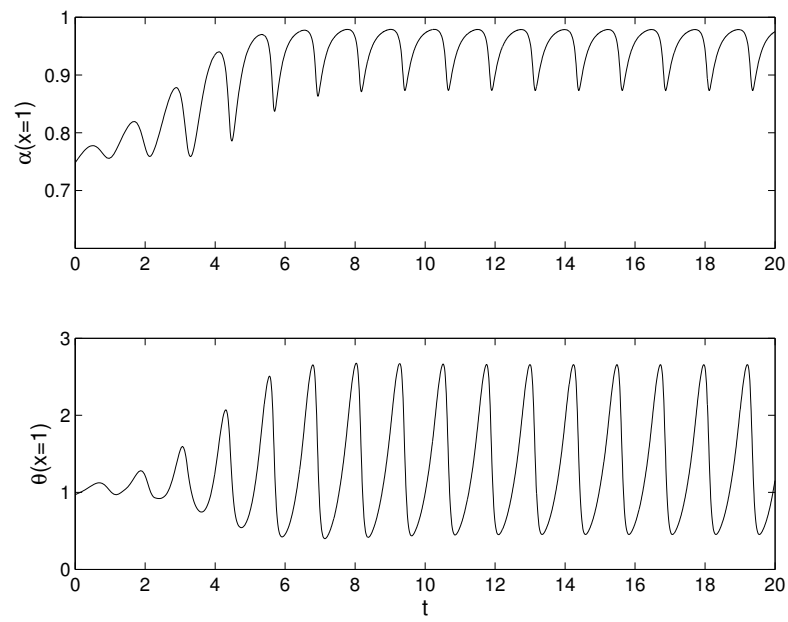

Fig. 1. Dynamic behavior of the outlet conversion and temperature of the adiabatic fixed-bed reactor with feed-effluent heat-exchange.

large systems the Householder operations in Algorithm 2 becomes the computational bottleneck and limits the system size to which the proposed continuous-discrete extended Kalman filter is practical.

For comparison, consider a standard EKF integrating the mean-covariance pair (2) directly. Only the equations corresponding to the lower triangular part of the symmetric covariance matrix are integrated. The integrator applied is Matlab's ODE15s [30] and the linear algebra operations of the EKF are implemented in a direct fashion without numerical stability considerations (i.e. without using orthogonal operations). The Jacobian of (2) for the fixed-bed reactor is very sparse. Furthermore, the sparsity pattern is irregular and narrows due to the fact that only the lower triangular part of the covariance matrix is actually included in the model. It should be noted that this Jacobian is very sparse and of much larger size than the Jacobian of the system model without the covariance matrix. The Jacobian of (2) is obtained numerically, as its analytic derivation for the fixedbed reactor would be very tedious.

Table I shows CPU times for standard EKF implementation and the EKF implementation based on ESDIRK34 ${ }^{1}$. The tests are conducted without process and measurement noise. The initial state estimate is equal to the nominal value. As the random variables are equal to their mean, this ensures that all cases go through the scenario depicted in Figure 1 and that the cases are comparable. The system is integrated from $t=0$ to $t=20$ and sampled every $T_{s}=0.2$ time unit. This implies that 100 integrations are conducted in each test case. The average time to do one extended Kalman filter computation, i.e. integrate $T_{s}=0.2$ time units ahead and do the linear algebra operations, are reported in Table I. Even for systems of relative small size $(N=25$ and $N=30)$, the extended Kalman filter algorithm reported in this paper is about two orders of magnitude faster than the standard implementation of the extended Kalman filter. The standard EKF is based on ODE15s while the ESDIRK34-EKF is based on ESDIRK. The better performance of the ESDIRK34-EKF is not due to ESDIRK in itself being a better integrator than ODE15s. This can be seen by comparing the integration times without covariance computations. In that respect ODE15s performs marginally better than ESDIRK34 ${ }^{2}$. Based on Table I and algorithmic profiling it is concluded, that the extra computing time needed of ESDIRK34-EKF compared to ESDIRK34 is mainly due to the time spent in Algorithm 2 computing the square root of the covariance matrix and only marginally due to the algebraic operations in Algorithm 3. For small systems $(N \leq 50)$ the time needed by ESDIRK34-EKF is about 2-5 times the time needed to integrate the system itself by ESDIRK34. However, for large systems the Householder operations in Algorithm 2 dominates the overall computing time as seen for $N=100$ and $N=200$ in Table I. As the Householder operations scale cubically with $n=2 N$, this limits the system size to which the proposed extended Kalman filter algorithm can practically be applied. However, it is about two orders of magnitude faster than the standard extended Kalman filter for small to moderate sized systems.

\section{CONCLUSION}

A computational efficient and robust algorithm for the extended Kalman filter for stochastic continuous-discrete

\footnotetext{
${ }^{1}$ All simulations are conducted using Matlab 7.0 on a Pentium IV 3.20 $\mathrm{GHz}$ processor.

${ }^{2}$ ESDIRK34 applies the same integrator as used in ESDIRK34-EKF. This integrator evaluates the Jacobian at each successful integration step. This is necessary for the presented EKF algorithm. However, for pure simulation applications, the Jacobian can be evaluated adaptively, making the ESDIRK34 algorithm performance comparable to the performance of ODE15s. Hence, based on Table I one should not conclude that ODE15s in itself is a better integrator than ESDIRK34.
} 
TABLE I

CPU-TIME (SEC.) FOR VARIOUS EKF IMPLEMENTATIONS FOR THE FIXED-BED REACTOR WITH FEED-EFFLUENT HEAT-EXCHANGE. THE CPU-TIME IS THE AVERAGE FOR ADVANCING 0.2 IN TIME IN THE INTERVAL 0 TO 20 USING THE LIMIT CYCLE AS NOMINAL SOLUTION. PENTIUM IV 3.20 GHZ AND MATLAB 7.0. atol $=10^{-6}$ AND $r t o l=10^{-3}$.

\begin{tabular}{c|c|c|c|c|c|c|c|c|c}
\hline & & \multicolumn{2}{|c|}{ Standard EKF } & \multicolumn{2}{c}{ ESDIRK34-EKF } & \multicolumn{2}{c|}{ ESDIRK34 } & \multicolumn{2}{c}{ ODE15s } \\
\hline$N$ & $n=2 N$ & Dense & Sparse & Dense & Sparse & Dense & Sparse & Dense & Sparse \\
\hline 25 & 50 & 13.43 & 8.49 & 0.12 & 0.15 & 0.07 & 0.08 & 0.05 & 0.05 \\
30 & 60 & 37.10 & 19.01 & 0.18 & 0.22 & 0.07 & 0.08 & 0.06 & 0.06 \\
40 & 80 & - & - & 0.31 & 0.40 & 0.11 & 0.09 & 0.07 & 0.06 \\
50 & 100 & - & - & 0.50 & 0.62 & 0.13 & 0.11 & 0.08 & 0.07 \\
100 & 200 & - & - & 4.46 & 4.43 & 0.37 & 0.22 & 0.17 & 0.10 \\
200 & 400 & - & - & 38.86 & 29.32 & 1.47 & 0.48 & 0.59 & 0.17 \\
\hline
\end{tabular}

systems has been presented. It is based on efficient integration of the state-covariance pair of the systems of stochastic differential equations using an ESDIRK algorithm with state sensitivity capabilities. For large scale systems it is more than two orders of magnitude faster than current standard implementations of the extended Kalman filter. This feature expands grey-box modelling of stochastic differential equation systems to large-scale systems. Furthermore, the computational efficiency is important in nonlinear model predictive control applications.

Application of state sensitivity computations to the extended Kalman filter algorithm has been demonstrated. The procedure presented is based on the ESDIRK algorithm which is well-suited for stiff problems with relative short measurement intervals. This is typically the case for most control applications of the extended Kalman filter. For nonstiff problems, explicit integration methods that do not need inversion of the iteration matrix would be more efficient than the presented method based on ESDIRK.

\section{REFERENCES}

[1] A. H. Jazwinski, Stochastic Processes and Filtering Theory. Academic Press, 1970.

[2] P. S. Maybeck, Stochastic Models, Estimation, and Control. London: Academic Press, 1982.

[3] W. H. Sorenson, Ed., Kalman Filtering: Theory and Application. London: IEEE Press, 1985.

[4] T. Kailath, A. H. Sayed, and B. Hassibi, Linear Estimation. Prentice Hall, 2000.

[5] S.-S. Jang, B. Joseph, and H. Mukai, "Comparison of two approaches to on-line parameter and state estimation of nonlinear systems," Ind. Eng. Chem. Process Des. Dev., vol. 25, pp. 809-814, 1986.

[6] C. V. Rao and J. B. Rawlings, "Nonlinear moving horizon state estimation," in Nonlinear Model Predictive Control, F. Allgöwer and A. Zheng, Eds. Basel: Birkhäuser, 2000, pp. 45-69.

[7] C. V. Rao, J. B. Rawlings, and J. H. Lee, "Constrained linear state estimation - a moving horizon approach," Automatica, vol. 37, pp. 1619-1628, 2001.

[8] E. L. Haseltine and J. B. Rawlings, "A critical evaluation of extended kalman filtering and moving horizon estimation," TWMCC, TexasWisconsin Modeling and Control Consortium, Tech. Rep. TWMCC2002-03, March 2003.

[9] H. Melgaard, "Identification of physical models," Ph.D. dissertation, Technical University of Denmark, 1994.

[10] N. R. Kristensen, H. Madsen, and S. B. Jørgensen, "Parameter estimation in stochastic grey-box models," Automatica, vol. 40, pp. 225-237, 2004.

[11] L. Ljung, System Identification. Theory for the User, 2nd ed. Upper Saddle River, NJ: Prentice Hall, 1999.
[12] D. Dochain, "State and parameter estimation in chemical and biochemical processes: A tutorial," Journal of Process Control, vol. 13, pp. 801-818, 2003

[13] J. H. Lee and N. L. Ricker, "Extended kalman filter based nonlinear model predictive control," Ind. Eng. Chem. Res., vol. 33, p. 1530, 1994.

[14] J. Valappil and C. Georgakis, "Systematic estimation of state noise statistics for extended kalman filters," AIChE Journal, vol. 46, p. 292, 2000.

[15] — , "Nonlinear model predictive control of end-use properties in batch reactors," AIChE Journal, vol. 48, pp. 2006-2021, 2002.

[16] _ - "Accounting for batch reactor uncertainty in the nonlinear MPC of end-use properties," AIChE Journal, vol. 49, pp. 1178-1192, 2003.

[17] M. Diehl, I. Uslu, R. Findeisen, S. Schwarzkopf, F. Allgöwer, H. G. Bock, T. Bürner, E. D. Gilles, A. Kienle, J. P. Schlöder, and E. Stein, "Real-time optimization for large scale processes: Nonlinear model predictive control of a high purity distillation column," in Online Optimization of Large Scale Systems: State of the Art, M. Grötschel, S. O. Krumke, and J. Rambau, Eds. New York: Springer, 2001.

[18] Z. K. Nagy and R. D. Braatz, "Robust nonlinear model predictive control of batch processes," AIChE Journal, vol. 49, pp. 1776-1786, 2003

[19] H. Bechmann, H. Madsen, N. K. Poulsen, and M. K. Nielsen, "Grey box modelling of first flush and incoming wastewater at a wastewater treatment plant," Environmetrics, vol. 11, pp. 1-12, 2000.

[20] N. R. Kristensen, H. Madsen, and S. B. Jørgensen, "A method for systematic improvement of stochastic grey-box models," Computers and Chemical Engineering, vol. 28, pp. 1431-1449, 2004.

[21] K. J. Åström, Introduction to Stochastic Control Theory. New York: Academic Press, 1970.

[22] P. E. Kloeden and E. Platen, Numerical Solution of Stochastic Differential Equations. Berlin: Springer, 1995.

[23] N. R. Kristensen, "Fed-batch process modelling for state estimation and optimal control," Ph.D. dissertation, Technical University of Denmark, 2002.

[24] R. Alexander, "Design and implementation of DIRK integrators for stiff systems," Applied Numerical Mathematics, vol. 46, pp. 1-17, 2003.

[25] M. R. Kristensen, J. B. Jørgensen, P. G. Thomsen, and S. B. Jørgensen, "An ESDIRK method with sensitivity analysis capabilities," Computers and Chemical Engineering, vol. 28, pp. 2695-2707, 2004.

[26] M. Schlegel, W. Marquardt, R. Ehrig, and U. Nowak, "Sensitivity analysis of linearly-implicit differential-algebraic systems by one-step extrapolation," Applied Numerical Mathematics, vol. 48, pp. 83-102, 2004.

[27] M. Caracotsios and W. E. Stewart, "Sensitivity analysis of initial value problems with mixed ODEs and algebraic equations," Computers and Chemical Engineering, vol. 9, pp. 359-365, 1985.

[28] W. J. Rugh, Linear System Theory, 2nd ed. Upper Saddle River, New Jersey: Prentice Hall, 1996.

[29] Y. Liu and E. W. Jacobsen, "On the use of reduced order models in bifurcation analysis of distributed parameter systems," Computers and Chemical Engineering, vol. 28, pp. 161-169, 2004.

[30] L. F. Shampine, M. W. Reichelt, and J. A. Kierzenka, "Solving index-1 DAEs in MATLAB and simulink," SIAM Review, vol. 41, pp. 538-552, 1999. 